\title{
AN ELEMENTARY PROOF OF A FINITE RIGIDITY PROBLEM BY INFINITESIMAL RIGIDITY METHODS ${ }^{1}$
}

\author{
EDGAR KANN
}

DEDICATED TO TAKIO MINAGAWA

\begin{abstract}
Let two compact, isometric surfaces with boundary be given having positive gauss curvature. If the surfaces can be placed so that their normal spherical images lie in a compact subset of a hemisphere of the unit sphere and so that the isometry is the identity on the boundary then the isometry is the identity mapping.

The proof is elementary in the sense that no integral formulae or maximum principles for elliptic operators are needed.

An example is given of a surface satisfying the above hypotheses which is neither convex nor has a representation in the form $z=f(x, y)$.
\end{abstract}

1. Introduction. The purpose of this paper is to provide an elementary proof by methods of infinitesimal rigidity of the following result:

Let two compact, isometric surfaces with boundary be given having positive gauss curvature. If the surfaces can be placed so that their normal spherical images lie in the same hemisphere of the unit sphere and so that the isometry is the identity on the boundary, then the isometry is the identity mapping.

The proof is elementary in the sense that no integral formulae or maximum principles for elliptic operators are needed. The facts quoted from [2] in $\$ 3$ are derived from straightforward calculations such as occur in a basic text in classical differential geometry of surfaces, for example [4]. However, it should be noted that surfaces satisfying the above hypotheses need not be convex nor have a representation in the form $z=f(x, y)$ so that certain standard methods could not be used, at least in their simplest form (see $\S 5$ ).

Infinitesimal methods have been used by Cohn-Vossen [1] to prove the unique determination of an ovaloid by its metric and by Pogorelov [5] to prove the general monotypy theorem for convex surfaces.

The infinitesimal methods enter as follows: If $X^{\prime}$ and $X^{\prime \prime}$ are position vectors of two isometric surfaces then the condition $d X^{\prime 2}=d X^{\prime \prime 2}$ is equivalent to $d X \cdot d Z=0$ where $X=\frac{1}{2}\left(X^{\prime \prime}+X^{\prime}\right)$ and $Z=\frac{1}{2}\left(X^{\prime \prime}-X^{\prime}\right)$. If $Z$ is

Received by the editors November 11, 1974 and, in revised form, March 24. 1976.

AMS (MOS) subject classifications (1970). Primary 53A05, 53C45; Secondary 53C40.

Key words and phrases. Infinitesimal rigidity, isometric surfaces, congruent surfaces, rotation vector.

${ }^{1}$ This paper contains the results of research done at Waseda University, Tokyo and at Queens College of the City University of New York under the support of grant number 1052 from the City University of New York Research Program. 
interpreted as a deformation field on the "mean surface" $X$ then $d X \cdot d Z=0$ is just the condition that $Z$ be an infinitesimal bending field on the surface $X$. The methods developed in [3] are then used to show that $Z$ is trivial.

It would be nice to prove the uniqueness theorem for ovaloids by the present methods. The difficulty is that the mean surface need not be regular for certain positions of the surfaces in space.

The author would like to express his thanks to the members of the Department of Mathematics, School of Education of Waseda University for their hospitality during his stay there and also to Professor Louis Nirenberg for his advice and encouragement.

2. Precise formulation of the problem. All manifolds will be of class $C^{\prime \prime}$. A manifold with boundary will be assumed to be immersed in a manifold. Likewise when we speak of a manifold with compact closure $\bar{M}$, it will be understood that $\bar{M}$ is immersed in a manifold. Any map $f$ on such objects is to be understood as a restriction from the containing manifold.

Let $M$ be a compact two-dimensional, orientable Riemannian manifold with boundary, having gauss curvature $K>0$.

Let $f^{\prime}: M \rightarrow E^{3}$ and $f^{\prime \prime}: M \rightarrow E^{3}$ be isometric immersions (also called surfaces), i.e., locally one-one mappings of $M$ in $E^{3}$ of rank two such that the metric induced on the images by $E^{3}$ equals the metric of $M$.

Represent the surfaces by position vectors $X^{\prime}=f^{\prime}(x)$ and $X^{\prime \prime}=f^{\prime \prime}(x)$ for $x \in M$. Then $d X^{\prime 2}=d X^{\prime \prime 2}$ at $x$.

To fix our ideas we suppose the unit surface normals $n^{\prime}$ and $n^{\prime \prime}$ are taken to point toward the concave sides of the surfaces.

Main Result. If

1. $f^{\prime}(x)=f^{\prime \prime}(x)$ for $x \in \partial M$,

2. there exists a constant vector $e$ such that $e \cdot n^{\prime}>0$ and $e \cdot n^{\prime \prime}>0$, then $f^{\prime}(x) \equiv f^{\prime \prime}(x)$ for $x \in M$.

3. General considerations of infinitesimal bending theory. We here follow Efimov [2, pp. 53-57]. Let $Z$ be a $C^{\prime \prime}$ vector field defined on a surface $S$ in $E^{3}$.

Definition. $Z$ is a bending field on $S$ if $d Z \cdot d X=0$.

If $u, v$ are local parameters on $S$ this is equivalent to the differential equations

$$
X_{u} \cdot Z_{u}=0, \quad X_{u} \cdot Z_{v}+X_{v} \cdot Z_{u}=0, \quad X_{v} \cdot Z_{v}=0,
$$

where $X_{u}=\partial X / \partial u$, etc. This is equivalent to the fact that the first fundamental form of the family of surfaces $X(u, v, t)$ is constant to first order in $t$ at $t=0$, under suitable differentiability hypotheses (with $Z=\partial X / \partial t$ at $t=0$ ).

It is known that if the surface is regular then a unique $C^{\prime}$ vector field $Y$ (called the rotation field) exists such that $Y \times d X=d Z$, or equivalently,

$$
Y \times X_{u}=Z_{u}, \quad Y \times X_{v}=Z_{v} .
$$

Further, there exist scalars $\alpha, \beta, \gamma$ such that 


$$
Y_{u}=\alpha X_{u}-\beta X_{v}, \quad Y_{v}=\gamma X_{u}-\alpha X_{v}
$$

If $Z$ is taken as a position vector it describes a surface called the bending surface. Likewise $Y$ describes the rotation surface. Both of these surfaces may be singular even when $S$ is everywhere regular, which is the usual assumption in infinitesimal bending theory. In the present applications this assumption is not made for $S$. At singular points of $S, Y$ is not defined.

A bending field $Z$ is said to be trivial if it is of the form $Z=C \times X+D$ where $C, D$ are constant vectors. Thus a trivial bending field coincides with the velocity field of a rigid motion of $S$.

Two bending fields which differ by a trivial bending field are called equivalent.

If a surface admits only trivial bendings it is called infinitesimally rigid.

\section{Proof of Main Result.}

LemMa 1. Let $M$ be a two-dimensional orientable manifold with compact closure $\bar{M}$. Let $f: \bar{M} \rightarrow E^{3}$ be an immersion of $\bar{M}$ such that: $E^{3}$

1. $f(\bar{M})$ is a surface having gauss curvature $K>0$ in the metric induced by

2. There exists a constant vector $e$ such that $e \cdot n>0$, where $n$ is the unit surface normal of $f(\bar{M})$ (hemisphere condition);

3. $A$ bending field $Z$ exists on $f(\bar{M})$ with corresponding rotation field $Y$.

Then $Y \cdot n \neq 0$ on the frontier of $M$ implies $Y \cdot n \neq 0$ on $M$.

Proof. First we prove the lemma under the assumption that $Y \neq 0$ on $M$. Suppose, by way of contradiction, that there existed a point $x$ of $M$ at which $Y \cdot n=0$. Not both $(Y \cdot n)_{u}=0$ and $(Y \cdot n)_{v}=0$ at $x$, for, by (3.2), it follows that $Y \cdot n_{u}=0$ and $Y \cdot n_{v}=0$. Since $K>0, n_{u}, n_{v}$ and $n$ are linearly independent, so $Y$ would vanish.

Hence, the implicit function theorem implies that an open, smooth $C^{\prime \prime}$ curve segment exists on $M$, containing $x$, on which $Y \cdot n=0$. The endpoints of the segment also lie in $M$ by hypothesis and the argument can be applied again to extend the segment indefinitely in both directions to a simple curve $\sigma$ which stays away from the frontier of $M$ (i.e., is contained in a compact subset of $M$ ).

$f(\sigma)$ may be represented by a vector $X=X(s),-\infty<s<+\infty$, where $s$ is arclength.

Since $K>0,(d X / d s) \cdot(d n / d s) \neq 0$. This, together with $Y \cdot d n / d s=0$, implies that $Y \times d X / d s \neq 0$ on $\sigma$.

Let $\lambda$ be defined on $\sigma$ by $Y \times d X / d s=\lambda n$. $\lambda$ never vanishes and simple compactness arguments show that $\lambda$ and $e \cdot n$ are bounded away from zero on o. Hence

$$
\frac{d(e \cdot Z)}{d s}=\frac{d Z}{d s} \cdot e=Y \times \frac{d X}{d s} \cdot e=\lambda e \cdot n \gg 0 .
$$

Thus $e \cdot Z$ would be unbounded on the compact set $\bar{M}$. Thus the assumption that $Y \cdot n=0$ leads to a contradiction. 
We conclude the proof of Lemma 1 by showing that if $Y$ is any rotation vector satisfying the hypothesis, then a rotation vector exists which satisfies the same hypothesis but never vanishes.

The spherical image mapping, $n: f(\bar{M}) \rightarrow U=$ the unit sphere, takes $\bar{M}$ to the compact subset of the open hemisphere of $U$ defined by hypothesis 2 , i.e., $\{X \in U: e \cdot X>0\}$. Hence if $g$ is any nonzero vector sufficiently close in direction to $e$ then $(n \circ f) \bar{M}$ lies in the hemisphere $\{X \in U: g \cdot X>0\}$. In other words there exists an open solid half-cone $C$ of vectors $g$ such that $g \cdot n>0$ where $n=(n \circ f) x$ for $x \in \bar{M}, g \in C$.

By hypothesis, $Y \cdot n \neq 0$ on the frontier of $M$. Suppose for example $Y \cdot n>0$ there. Since the frontier of $M$ is compact, $Y \cdot n$ has a positive minimum, $m$, there.

The rotation surface $R$ described by the position vector $Y$ cannot contain an open subset of $E^{3}$. Hence there exists a constant vector $g_{0} \in C$ such that $Y-g_{0} \neq 0$ for any $Y \in R$ and such that $\left|g_{0}\right|<m$. Hence we have $Y \cdot n \geqslant$ $m>g_{0} \cdot n>0$ on the frontier of $M$.

It follows that $Y-g_{0}$ is a rotation vector for the bending field $Z-g_{0} \times X$ (equivalent to $Z$ ) such that $\left(Y-g_{0}\right) \cdot n>0$ on the frontier of $M$. By the first part of our proof $\left(Y-g_{0}\right) \cdot n>0$ on $\bar{M}$. Hence $Y \cdot n>0$ on $\bar{M}$.

Similarly the assumption $Y \cdot n<0$ on the frontier of $M$ leads to $Y \cdot n<0$ on $\bar{M}$.

NoTE. The proof yields a somewhat stronger conclusion: If $Y \cdot n \gtrless 0$ on the frontier of $M$ then there exists a vector $g_{0}$ such that $Y \cdot n \gtrless g_{0} \cdot n \geqslant 0$ on $M$.

LEMma 2. Let the hypothesis be as in Lemma 1 except that $f$ need not be of rank 2 on the frontier of $M$. Then $Z_{u} \times Z_{v} \neq 0$ on the frontier of $M$ implies $Z_{u} \times Z_{v} \neq 0$ on $M$.

Proof. At any point of $M$ (but not necessarily on the frontier of $M$, since $Y$ need not be defined there)

$$
Z_{u} \times Z_{v}=\left(Y \times X_{u}\right) \times\left(Y \times X_{v}\right)=\left(Y \cdot X_{u} \times X_{v}\right) Y .
$$

Thus $Z_{u} \times Z_{v}=0$ on $M$ if and only if $Y \cdot n=0$.

By continuity of $Z_{u} \times Z_{v}$ there is a closed neighborhood $N$ of the frontier of $M$ such that $Z_{u} \times Z_{v} \neq 0$ there and such that the hypotheses of Lemma 1 are satisfied by $Y \cdot n$ on $\bar{M}-\bar{M} \cap N$.

Proof of Main Result. Define $X=\frac{1}{2}\left(X^{\prime}+X^{\prime \prime}\right), Z=\frac{1}{2}\left(X^{\prime \prime}-X^{\prime}\right)$. As mentioned in the introduction, $d X \cdot d Z=0$, so $Z$ is a bending field on the surface $X$, which may be singular.

$$
\begin{aligned}
X_{u} \times X_{v} & =\frac{1}{2}\left(X_{u}^{\prime}+X_{u}^{\prime \prime}\right) \times \frac{1}{2}\left(X_{v}^{\prime}+X_{v}^{\prime \prime}\right) \\
& =\frac{1}{4}\left(X_{u}^{\prime} \times X_{v}^{\prime}+X_{u}^{\prime \prime} \times X_{v}^{\prime}+X_{u}^{\prime} \times X_{v}^{\prime \prime}+X_{u}^{\prime \prime} \times X_{v}^{\prime \prime}\right),
\end{aligned}
$$

and 


$$
\begin{aligned}
Z_{u} \times Z_{v} & =\frac{1}{2}\left(X_{u}^{\prime \prime}-X_{u}^{\prime}\right) \times \frac{1}{2}\left(X_{v}^{\prime \prime}-X_{v}^{\prime}\right) \\
& =\frac{1}{4}\left(X_{u}^{\prime} \times X_{v}^{\prime}-X_{u}^{\prime \prime} \times X_{v}^{\prime}-X_{u}^{\prime} \times X_{v}^{\prime \prime}+X_{u}^{\prime \prime} \times X_{v}^{\prime \prime}\right),
\end{aligned}
$$

so

$$
X_{u} \times X_{v}+Z_{u} \times Z_{v}=\frac{1}{2}\left(X_{u}^{\prime} \times X_{v}^{\prime}+X_{u}^{\prime \prime} \times X_{v}^{\prime \prime}\right)
$$

hence

$$
e \cdot X_{u} \times X_{v}+e \cdot Z_{u} \times Z_{v}>0 \text { on } M
$$

follows from hypothesis 2 . But $e \cdot X_{u} \times X_{v}>0$ on $\partial M$ since $Z_{u} \times Z_{v}=0$ there. Hence there is an open set $U$ of $M$ such that $e \cdot X_{u} \times X_{v}>0$ on $U$ and $U$ is adjacent to $\partial M$, that is, part of the frontier of $U$ is $\partial M$. Thus $X$ is regular on $U$.

Let $U^{*}$ be the maximal open subset of $M$ on which $e \cdot X_{u} \times X_{v}>0$. The frontier of $U^{*}$ is the union of two disjoint sets, $\partial M$ and a set $A$ on which $e \cdot X_{u} \times X_{v}=0$. $A$ may be empty, in which case $U^{*}=M$.

Define $Z_{\lambda}=Z+\lambda e \times X$ where $\lambda$ is an arbitrary real number. $Z_{\lambda}$ is a bending field on $X$ equivalent to $Z$.

$$
\begin{aligned}
\left(Z_{\lambda}\right)_{u} \times\left(Z_{\lambda}\right)_{v}= & \left(Z_{u}+\lambda e \times X_{u}\right) \times\left(Z_{v}+\lambda e \times X_{v}\right) \\
= & Z_{u} \times Z_{v} \\
& +\lambda\left[\left(Z_{u} \cdot X_{v}\right) e-\left(Z_{u} \cdot e\right) X_{v}+\left(Z_{v} \cdot e\right) X_{u}-\left(Z_{v} \cdot X_{u}\right) e\right] \\
& +\lambda^{2}\left(e \cdot X_{u} \times X_{v}\right) e .
\end{aligned}
$$

The linear dependence of $Z_{u}$ and $Z_{v}$ on $\partial M$ together with (3.0) imply that on $\partial M$,

$$
\left(Z_{\lambda}\right)_{u} \times\left(Z_{\lambda}\right)_{v}=\lambda\left[\left(Z_{v} \cdot e\right) X_{u}-\left(Z_{u} \cdot e\right) X_{v}\right]+\lambda^{2}\left(e \cdot X_{u} \times X_{v}\right) e .
$$

Now $X_{u}, X_{v}, e$ are linearly independent on $\partial M$ so

$$
\left(Z_{\lambda}\right)_{u} \times\left(Z_{\lambda}\right)_{v} \neq 0 \text { on } \partial M \text { if } \lambda \neq 0
$$

From (4.0) it follows that on $A, Z_{u} \times Z_{v} \neq 0$. Since $A$ is compact $\mid Z_{u} \times$ $Z_{v} \mid \gg 0$ on $A$. Since $\left(Z_{\lambda}\right)_{u} \times\left(Z_{\lambda}\right)_{v}$ is a uniformly continuous function of $\lambda$ on $A$ it follows that $\left(Z_{\lambda}\right)_{u} \times\left(Z_{\lambda}\right)_{v} \neq 0$ on $A$ for $|\lambda|$ sufficiently small. Hence, by Lemma 2,

$$
\left(Z_{\lambda}\right)_{u} \times\left(Z_{\lambda}\right)_{v} \neq 0 \text { on } U^{*} \text { for small }|\lambda| \text { if } \lambda \neq 0 .
$$

$U^{*} \cup \partial M$ is a regular surface with boundary, represented by the position vector $X$, so a unique rotation vector $Y$ corresponding to the bending field $Z$ exists such that $Y \times d X=d Z$. Since

$$
d Z_{\lambda}=d Z+\lambda e \times d X=(Y+\lambda e) \times d X,
$$

$Y+\lambda e$ is the unique rotation vector for $Z_{\lambda}$.

$$
\begin{aligned}
\left(Z_{\lambda}\right)_{u} \times\left(Z_{\lambda}\right)_{v} & =\left[(Y+\lambda e) \times X_{u}\right] \times\left[(Y+\lambda e) \times X_{v}\right] \\
& =\left[(Y+\lambda e) \cdot\left(X_{u} \times X_{v}\right)\right](Y+\lambda e) .
\end{aligned}
$$

By (4.3) the last term in square brackets, call it $q_{\lambda}$, does not vanish on $U^{*}$ if $|\lambda|$ is small but not zero. 
But $Y \cdot X_{u} \times X_{v}=0$ on $\partial M$ (put $\lambda=0$ in (4.1) and in (4.4)) so $q_{\lambda}$ is positive or negative on $\partial M$, and hence near $\partial M$, according as $\lambda$ is positive or negative.

Since $q_{\lambda}$ does not vanish on $U^{*}$ it also is positive or negative on all of $U^{*}$ according as $\lambda$ is. Hence, by continuity, $q_{0} \equiv 0$ on $U^{*}$.

This is equivalent to $Y \cdot n \equiv 0$, where $n=X_{u} \times X_{v} /\left|X_{u} \times X_{v}\right|$. It follows from (3.2) that $Y \cdot n_{u} \equiv 0$ and $Y \cdot n_{v} \equiv 0$.

Now it is known (cf. introduction) that the gauss curvature of the mean surface $X$ is positive at regular points if the gauss curvatures of $X^{\prime}$ and $X^{\prime \prime}$ are. Therefore $n, n_{u}, n_{v}$ are linearly independent, hence $Y \equiv 0$. By (3.1) $Z_{u} \equiv 0$ and $Z_{v} \equiv 0$ so $Z$ is constant on $U^{*}$. Since $Z=0$ on $\partial M, Z \equiv 0$ on $U^{*}$. By (4.0) $A$ is empty, hence $U^{*}=M$ and $Z \equiv 0$ on $M$.

5. An example of a nonconvex surface satisfying the hypotheses. There is a tubular surface constructed on the cylindrical helix which satisfies the conditions of $\$ 2$ but is neither convex nor has a representation of the form

$$
z=f(x, y) \text {. }
$$

Let

$$
X(t)=a(i \cos t+j \sin t+c t k)
$$

where $i, j, k$ are orthonormal basis vectors and $a$ and $c$ are positive constants. This is a vector representation of a helix lying on a cylinder of radius $a$. Let $X(v)$ be a representation of the helix in terms of arclength $v$ and let $v_{1}, v_{2}, v_{3}$ be the Frenet triple of the helix, and $r$ a positive constant. Then the surface

$$
X(u, v)=X(v)+r\left(v_{2}(v) \cos u+v_{3}(v) \sin u\right)
$$

is a tubular surface (or canal surface) whose cross-sections by the normal planes of the helix are circles of radius $r$ (cf. [4, p. 76, Exercise 6.7.4]).

Elementary calculation using the Frenet equations yields

$$
X_{u} \times X_{v}=r(1-r \kappa \cos u)\left(v_{3}(v) \sin u+v_{2}(v) \cos u\right)
$$

so the surface is regular if $r<1 / \kappa$, where $\kappa$ is the curvature of the helix. Also,

$$
k \cdot X_{u} \times X_{v}=r(1-r \kappa \cos u)\left(a\left(a^{2}+c^{2}\right)^{-1 / 2} \sin u\right)
$$

and the gauss curvature is $K=-\kappa \cos u / \sqrt{g}$. Thus if we restrict $u$ to satisfy $\pi+\varepsilon \leqslant u \leqslant \frac{3}{2} \pi-\varepsilon$ where $\varepsilon$ is a small positive number we obtain a surface whose closure has positive gauss curvature and has its spherical image in a hemisphere. If $v$ is restricted to a closed interval then all of the conditions of $\$ 2$ are satisfied. Such a surface is clearly not convex and cannot be represented in the form $z=f(x, y)$ if the interval in which $v$ lies is sufficiently large.

\section{BIBLIOGRAPHY}

1. S. E. Cohn-Vossen, Verbiegbarkeit von Flächen im Grossen, Fortschritte Math. 1 (1936), 33-76. 
2. N. W. Efimov, Flächenverbiegung im Grossen, Akademie-Verlag, Berlin, 1957. MR 19, 59.

3. E. Kann, $A$ new method for infinitesimal rigidity of surfaces with $K>0$, J. Differential Geometry 4 (1970), 5-12. MR 41 \#4449.

4. D. Laugwitz, Differential and Riemannian goemetry, Academic Press, New York and London, 1965. MR 30 \#2406.

5. A. V. Pogorelov, Extrinsic geometry of convex surfaces, "Nauka", Moscow, 1969; English transl., Transl. Math. Monographs, vol. 35, Amer. Math. Soc., Providence, R. I., 1973. MR 39 \#6222; 49 \# 11439.

Department of Mathematics, Queens College (CUNY), Flushing, New York 11367 